\title{
Nosocomial Infections in Dialysis Access
}

\author{
Alexander Schweiger ${ }^{a} \cdot$ Sergio Trevino ${ }^{b} \cdot$ Jonas Marschall ${ }^{a}$ b

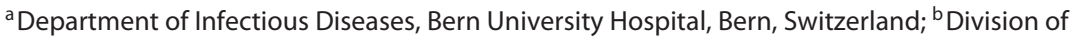 \\ Infectious Diseases, Washington University School of Medicine, St. Louis, Mo., USA
}

\begin{abstract}
Nosocomial infections in patients requiring renal replacement therapy have a high impact on morbidity and mortality. The most dangerous complication is bloodstream infection (BSI) associated with the vascular access, with a low BSI risk in arteriovenous fistulas or grafts and a comparatively high risk in central venous catheters. The single most important measure for preventing BSI is therefore the reduction of catheter use by means of early fistula formation. As this is not always feasible, prevention should focus on educational efforts, hand hygiene, surveillance of dialysis-associated events, and specific measures at and after the insertion of catheters. Core measures at the time of insertion include choosing the optimal site of insertion, the use of maximum sterile barrier precautions, adequate skin antisepsis, and the choice of catheter type; after insertion, access care needs to ensure hub disinfection and regular dressing changes. The application of antimicrobial locks is reserved for special situations. Evidence suggests that bundling a selection of the aforementioned measures can significantly reduce infection rates. The diagnosis of central lineassociated BSI (CLABSI) is based on clinical signs and microbiological findings in blood cultures ideally drawn both peripherally and from the catheter. The prompt installation of empiric antibiotic treatment covering the most commonly encountered organisms is key regarding CLABSI treatment. Catheter removal is recommended in complicated cases or if cultures yield Staphylococcus aureus, enterococci, Pseudomonas or fungi. In other cases, guide wire exchange or catheter salvage strategies with antibiotic lock solutions may be acceptable alternatives.




\section{Recommendations to Improve Patient Safety}

- The implementation of a multidisciplinary team and so-called prevention bundles can decrease infections and improve safety in dialysis patients.

- The most effective preventive measure for vascular access infections is using a fistula instead of a catheter.

- Surveillance of dialysis-associated events is necessary to develop and measure the effectiveness of preventive measures.

- Infectious Diseases Society of America and European Renal Best Practice have developed guidelines for the management of hemodialysis catheterassociated infections.

\section{Introduction}

Health care-associated infections (HAIs) are one of the major threats to patient safety, yet they have not been widely perceived as such before the advent of the patient safety movement. Between one and two third of HAIs are thought to be preventable [1], which would translate into ten thousands of saved lives and billions of dollars in expenses avoided in US hospitals alone each year. A number of entities such as the Centers for Disease Control and Prevention (CDC), the Society for Healthcare Epidemiology of America (SHEA), the European Renal Association (ERA), and also the patient safety-oriented Institute for Healthcare Improvement (www.ihi.org) therefore publish HAI prevention guidelines to advance implementation of best practices $[2,3]$. The incidence of central line-associated bloodstream infections (CLABSIs), which exhibit higher mortality than most other HAIs, may drop to rates as low as zero with a robust infection prevention program [4].

Hemodialysis (HD) catheter-associated bloodstream infections (BSIs) are a type of CLABSI due to a central venous catheter (CVC) specifically designed for HD. In 2002, it was estimated that 50,000 CLABSIs occur in dialysis patients in the US annually. A more recent surveillance study found the rate of access-related BSIs to be 0.73 events per 100 patient-months [5]. Aside from catheters, patients in need of renal replacement therapy should eventually use arteriovenous fistula (AVF) or arteriovenous grafts (AVG) for HD, or alternatively, may receive peritoneal dialysis. All renal replacement therapies are associated with a risk of infection; however, this risk varies with the method selected. Data from a CDC surveillance program display nicely how the type of access influences the BSI rate per 100 patient-months: 0.5 (for AVFs), 0.9 (AVG), 4.2 (permanent CVCs), and an impressive 27.1 for temporary CVCs [6]. Another, more practi- 
cal effect of an infection is that the vascular access is (at least temporarily) lost, thereby complicating care further. Therefore, the single most important preventive measure for CLABSI is using a fistula instead of a catheter. However, an AVF requires maturation before it can be accessed the first time. Temporary catheterization for HD may be necessary in cases where renal function deteriorates quickly and the fistula is not functional yet. An emphasis for early planning of vascular surgery has been made by the Centers for Medicare and Medicaid Services' Fistula First initiative. The goal of having a ready-to-use fistula in $66 \%$ of patients meeting dialysis criteria for the first time, however, has not yet been reached.

In the following text, we will discuss the current management and prevention strategies for HD catheter-associated BSIs. Because the population of patients requiring renal replacement therapy in the US is expected to grow from approximately 600,000 today to 750,000 in the year 2020 [7], implementing evidencebased infection prevention strategies now will spare our patients much suffering in the future.

\section{Prevention}

There is relatively little research on CLABSI in dialysis patients; nevertheless, several authorities have issued guidelines concerning the prevention of (dialysisassociated) CLABSI, including the CDC, SHEA and the ERA [2, 3, 8]. Major recommendations are summarized in table 1 . In addition, a bundled approach for prevention of BSI in dialysis facilities was proposed by the CDC [9]; key interventions are listed in table 2.

\section{Before Insertion}

\section{Staff Education and Competency}

All staff need to receive education upon entry into the facility, which has to be repeated in regular intervals (at least annually), and should be accompanied by regular audits to ensure sustained compliance. Education should focus on knowledge about nosocomial infections at dialysis centers, infection control best practices, including access care and aseptic technique, and how to educate patients and other caregivers [9]. 


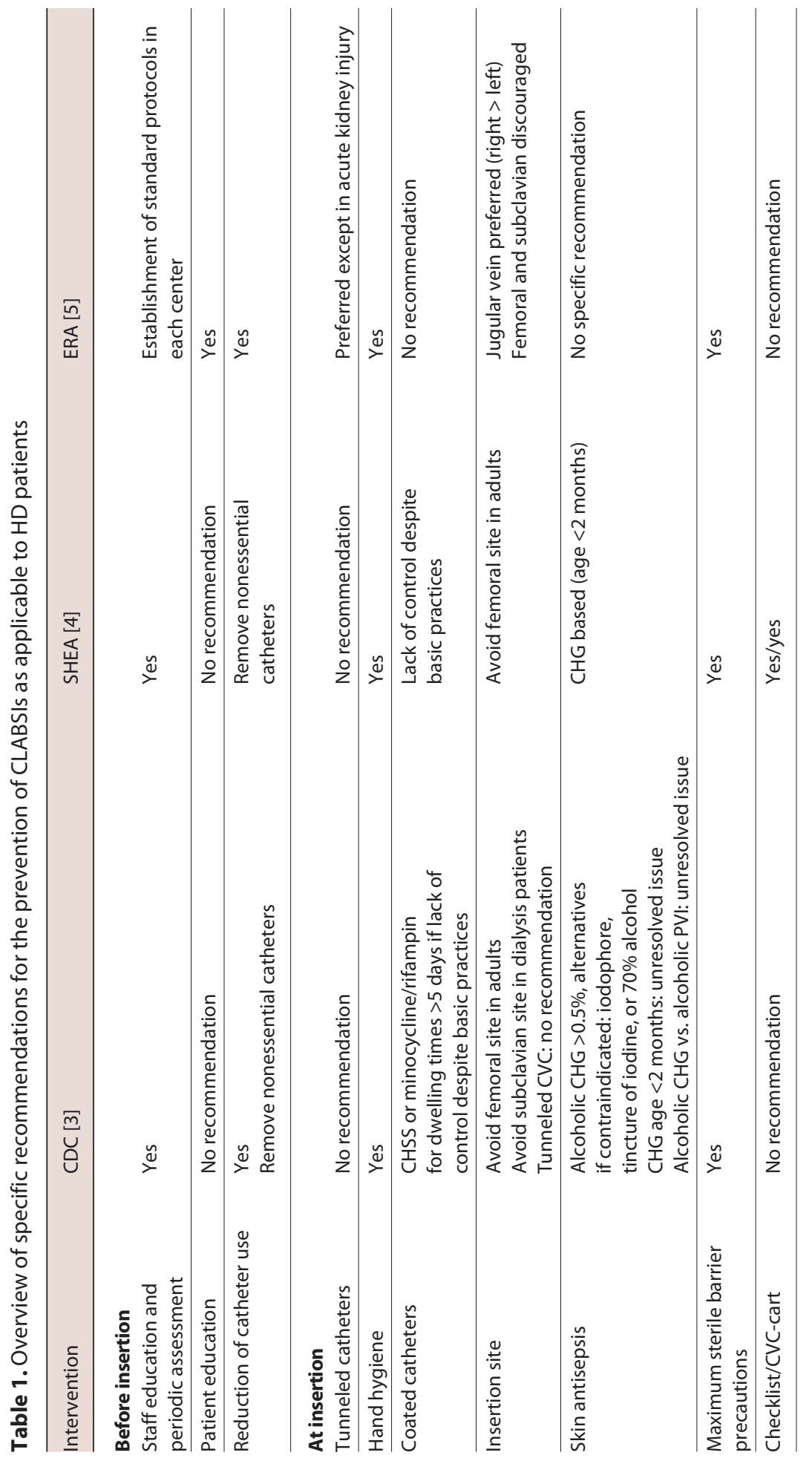




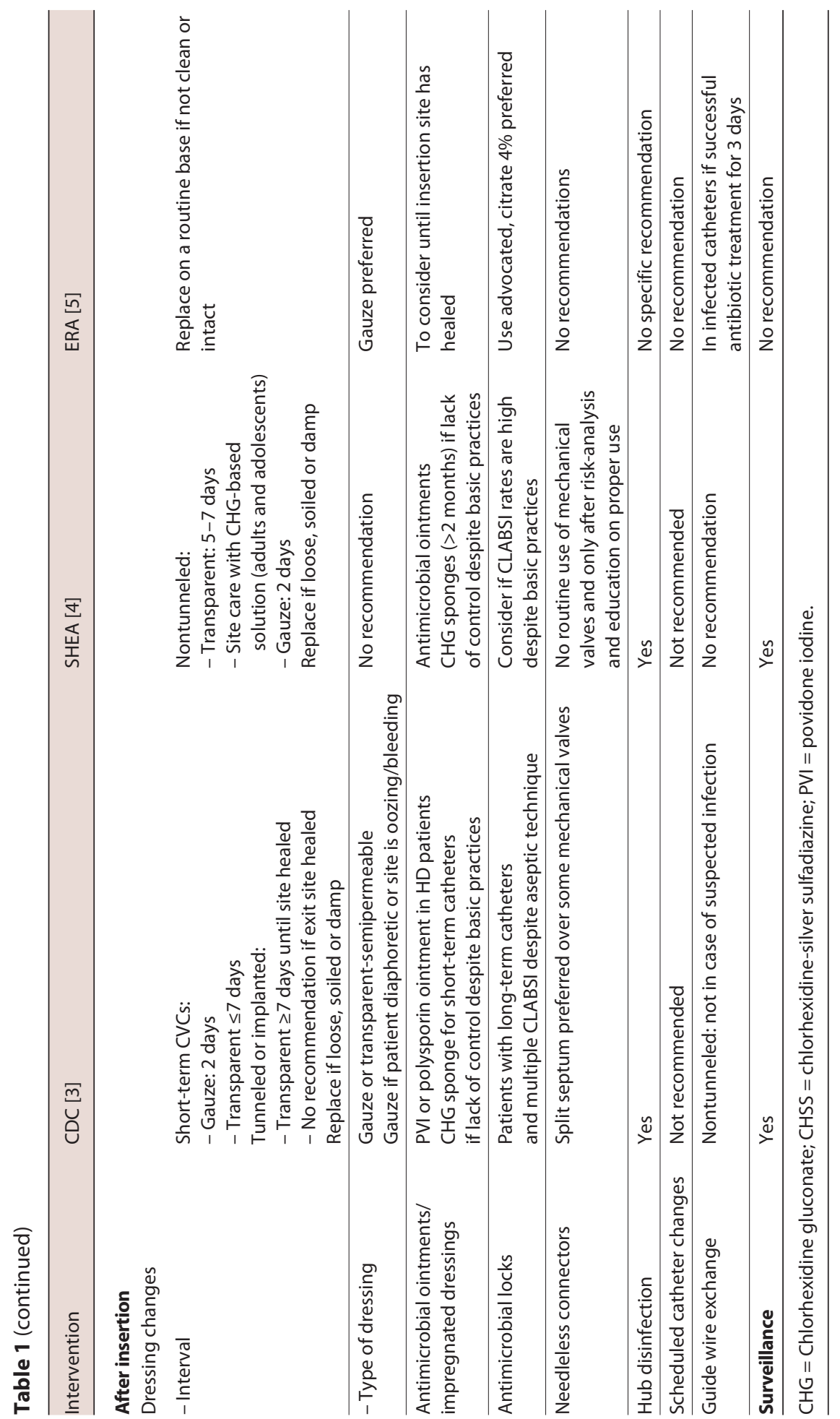


Table 2. Core interventions for dialysis BSI prevention [16]

\begin{tabular}{|c|c|}
\hline Key intervention & Contents \\
\hline Surveillance and feedback & $\begin{array}{l}\text { - Conduct monthly surveillance for BSIs and other } \\
\text { dialysis events using CDCs National Healthcare Safety } \\
\text { Network (NHSN) } \\
\text { - Calculate facility rates and compare to rates in other } \\
\text { NHSN facilities } \\
\text { - }\end{array}$ \\
\hline Hand hygiene observations & $\begin{array}{l}\text { - Perform observations of hand hygiene opportunities } \\
\text { monthly and share results with clinical staff }\end{array}$ \\
\hline $\begin{array}{l}\text { Catheter/vascular access } \\
\text { care observations }\end{array}$ & $\begin{array}{l}\text { - Perform observations of vascular access care and } \\
\text { catheter accessing quarterly } \\
\text { - Assess staff adherence to aseptic technique when } \\
\text { connecting and disconnecting catheters and during } \\
\text { dressing changes } \\
\text { - Share results with clinical staff }\end{array}$ \\
\hline $\begin{array}{l}\text { Staff education and } \\
\text { competency }\end{array}$ & $\begin{array}{l}\text { - Train staff on infection control topics, including access } \\
\text { care and aseptic technique } \\
\text { - Perform competency evaluation for skills such as } \\
\text { catheter care and accessing every } 6-12 \text { months and } \\
\text { upon hire }\end{array}$ \\
\hline $\begin{array}{l}\text { Patient education/ } \\
\text { engagement }\end{array}$ & $\begin{array}{l}\text { - Provide standardized education to all patients on } \\
\text { infection prevention topics including vascular access } \\
\text { care, hand hygiene, risks related to catheter use, } \\
\text { recognizing signs of infection, and instructions for } \\
\text { access management when away from the dialysis unit }\end{array}$ \\
\hline Reduction of catheter use & $\begin{array}{l}\text { - Incorporate efforts (e.g., through patient education, } \\
\text { vascular access coordinator) to reduce catheters by } \\
\text { identifying and addressing barriers to permanent } \\
\text { vascular access placement and catheter removal }\end{array}$ \\
\hline $\begin{array}{l}\text { Chlorhexidine for } \\
\text { skin antisepsis }\end{array}$ & $\begin{array}{l}\text { - Use an alcohol-based chlorhexidine }(>0.5 \%) \text { solution } \\
\text { as the first-line skin antiseptic agent for central line } \\
\text { insertion and during dressing changes } \\
\text { - Povidone-iodine (preferably with alcohol) or } 70 \% \\
\text { alcohol alternatives for patients with chlorhexidine } \\
\text { intolerance }\end{array}$ \\
\hline Catheter hub disinfection & $\begin{array}{l}\text { - Scrub catheter hubs with an appropriate antiseptic } \\
\text { after cap is removed and before accessing } \\
\text { - Perform every time catheter is accessed or } \\
\text { disconnected } \\
\text { - If closed needleless connector device is used, disinfect } \\
\text { connector device per manufacturer's instructions }\end{array}$ \\
\hline Antimicrobial ointment & $\begin{array}{l}\text { - Apply antibiotic ointment or povidone-iodine } \\
\text { ointment to catheter exit sites during dressing change } \\
\text { - Use of chlorhexidine-impregnated sponge dressing } \\
\text { might be an alternative }\end{array}$ \\
\hline
\end{tabular}




\section{Patient Education}

In its core interventions for BSI reduction, the CDC advocates patient education. Main components are (1) hand hygiene and basic infection control practices during catheter accessing process (e.g., aseptic technique, masks, hub disinfection), (2) access care at home, (3) role of and risks associated with catheters, and (4) knowing signs and symptoms of infection and when to notify the physician. Despite mixed results in other studies, Cheng et al. [10] described a $40 \%$ lower risk of hospitalization due to infection and a $51 \%$ mortality reduction in patients with chronic kidney disease who received a multidisciplinary education prior to dialysis onset. We conclude that patient education is a valuable tool in the prevention of CLABSIs.

\section{Hand Hygiene of Staff}

Hand washing, or preferably hand disinfection reduces HAIs in different settings, and is one of the key measures for preventing CLABSI. Regular audits on compliance with hand hygiene should be performed. Alcohol-based waterless products or the combination of antiseptic soap and water are preferred. The use of gloves does not obviate hand hygiene [11].

\section{At Insertion}

\section{Insertion Site}

HD catheters are inserted in the internal jugular vein (IJV) or femoral vein (FV). The subclavian site should be avoided due to the risk of stenosis formation, which may only manifest after AVF creation [3]. No significant differences in CLABSI rates were found between IJV and FV with short-term dialysis (median 5 days). But with longer dwelling time, the FV site carries a 5 times higher risk for bacteremia in dialysis patients than the IJV [12]. The likelihood of bacteremia for the FV site increases by catheterization weeks 1-2, whereas for the IJV the risk increase comes at a later time, around weeks 3-4. Both CDC and ERA recommend using the IJV first for HD.

In addition, maximum sterile barrier precautions for catheter insertion should be meticulously applied as they reduce the incidence of CLABSIs [4]. 


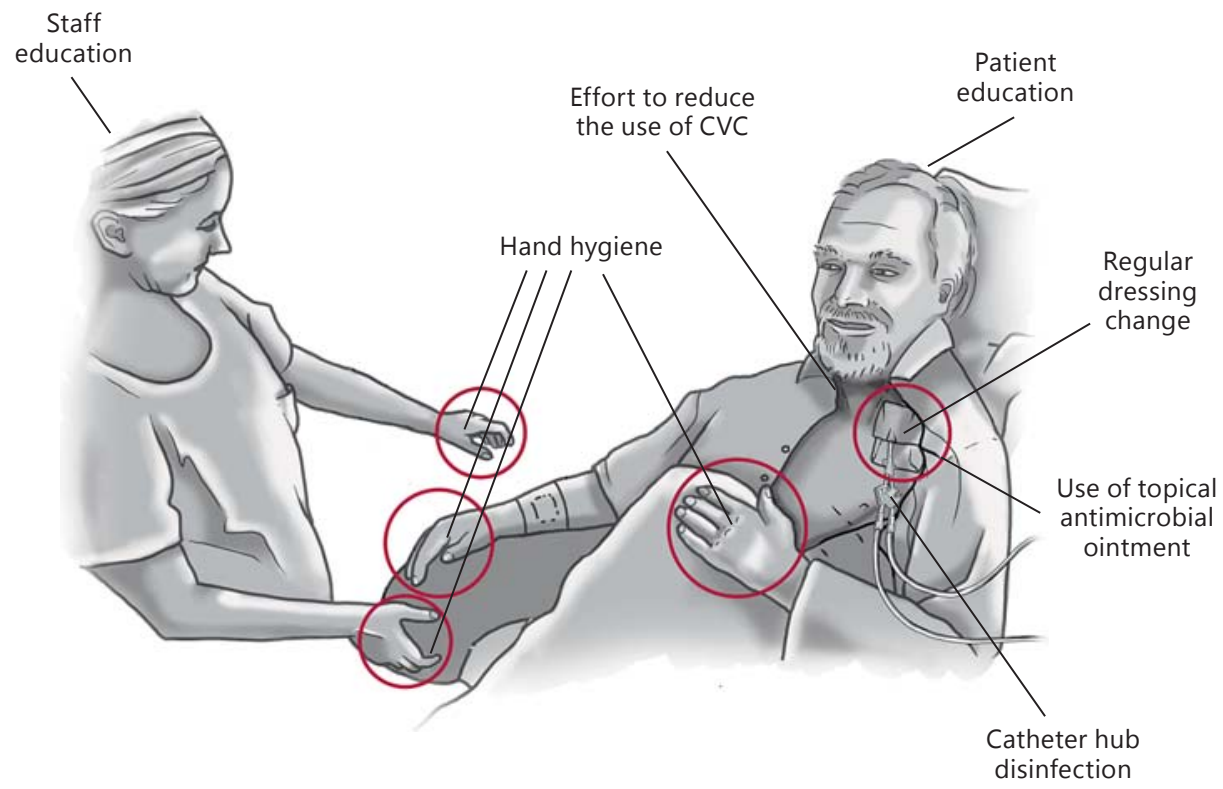

Fig. 1. Strategies to prevent CLABSIs.

\section{Coated Catheters}

The efficacy of antimicrobial coating of CVCs in the acute setting has been thoroughly evaluated. Coatings with chlorhexidine-silver sulfadiazine and minocycline/rifampin have been proven to significantly reduce the risk of CLABSI. In situations of high CLABSI rates and short-term dwelling times, these catheters can be cost-efficient. In HD patients, high-level evidence for significant reduction of the infection risk only exists for catheters coated with minocycline/rifampin [13]. Catheters solely coated with silver were not superior to untreated catheters in dialysis patients. Although some coated catheters will influence the CLABSI risk in dialysis patients, the additional cost has to be factored in.

\section{Skin Antisepsis}

Chlorhexidine gluconate (CHG) for skin antisepsis is superior to aqueous povidone iodine (PVI) for prevention of CLABSI [14]. In comparison to alcoholic PVI, no clear evidence exists as to the superiority or inferiority of CHG solutions. The optimal concentration of $\mathrm{CHG}$ remains unclear. The CDC advocate 
the use of alcoholic $\mathrm{CHG}>0.5 \%$, although this recommendation is mainly based on the fast bactericidal activity, longer-lasting antimicrobial effect, and the low rate of CHG inactivation by protein-rich materials (e.g., blood) [2]. Octenidine $0.1 \%$ in propranolol, which is not FDA approved, showed mixed results when compared with ethanol/propranolol or CHG. Further trials are needed to clarify its efficacy.

Whereas no reactions of polyurethane catheters to either of the aforementioned disinfectants are reported in the literature, PVI may adversely affect silicone tubing. Therefore, its compatibility with silicone catheters should be verified according to the manufacturer's recommendations prior to use.

\section{After Insertion}

As the risk of CLABSI increases in function of dwelling time $[12,15]$, catheter care after insertion is of paramount importance (fig. 1).

\section{Dressing Changes}

Dressing changes should be performed at regular intervals of up to 7 days for transparent dressings, which is not inferior to changes every 3 days [16]. Gauze dressings should be changed every other day, but with tunneled catheters the interval can be as long as 7 days [2]. Dressings should be changed earlier when loose, damp or soiled $[2,8]$. CHG-impregnated dressings or sponges significantly reduced the rate of CLABSIs when compared with standard dressings in nondialysis studies [16].

Camins et al. [17] evaluated the efficacy of CHG sponges (Biopatch ${ }^{\circledR}$ ) compared to standard of care (sodium-hypochlorite solution $0.114 \%$ ) in dialysis patients. No significant differences in terms of CLABSI rates were found. The authors suspected that the infection risk in their patient collective was more likely to have originated from the hub than from the insertion site.

The main adverse event of CHG is contact dermatitis ( $~ 5 \%$ rate), although $96 \%$ of these events occurred in neonates. Reduced susceptibility to CHG has been documented, but its clinical impact is unclear. In summary, the use of CHG-impregnated dressings reduced the CLABSI risk in short-term nontunneled CVCs, but as of yet no sound evidence supports its application in dialysis patients. 
Topical antimicrobial ointments (mupirocin, polysporin, PVI) can reduce CLABSI rates in HD patients. Specifically, mupirocin ointment significantly reduced CLABSIs (OR: 0.17, 95\% CI 0.07-0.43). No serious adverse events were reported in all studies included in this meta-analysis [18], nor are there reports of mupirocin resistance in studies involving dialysis patients. Polysporin antibiotic ointment (bacitracin/gramicidin/polymyxin B) was evaluated in a doubleblind, placebo-controlled, randomized trial including 162 dialysis patients, yielding a significantly lower rate of bacteremia [0.63 catheter-days (treatment group) vs. 2.48/1,000 (placebo group); $\mathrm{p}=0.0004$ ]. The time to first bacteremia, infection-related morbidity, and mortality rates also scored favorably in the treatment group [19]. Topical PVI significantly reduced CLABSI incidence as compared to sterile gauze alone ( 2 vs. $17 \%, \mathrm{p}<0.01$ ), and this risk reduction was most evident in nasal Staphylococcus aureus carriers [20]. Topical Medhoney (a medicinal honey formulation) did not reduce the risk of infectious complications [18].

In conclusion, evidence exists for the topical use of mupirocin, polysporin $B$, and to a lesser degree PVI for CLABSI prevention. The potential for mupirocin resistance development has to be considered. Currently, while gramicidin is not available in the US, a similar preparation in the form of bacitracin/neomycin/ polymyxin $B$ is commonly used.

\section{Hub Disinfection}

All hubs should be disinfected prior to and after accessing. The efficacy of different disinfection times $(3,10,15 \mathrm{~s})$ with $70 \%$ ethanol on deliberately S. aureuscontaminated hubs was evaluated. Although no significant difference in reduction of contamination was found, the number of colony-forming units was markedly lower after $15 \mathrm{~s}$. The authors posited a protecting role of longer disinfection [21]. No data exist for HD catheters.

More data are needed regarding the role of needleless connectors in the prevention of CLABSI. The safety of mechanical valve systems has been questioned in several studies on CLABSIs [22, 23]. If needleless connectors are used, splitseptum devices are preferred to devices with mechanical valves. Currently, there is no sound evidence supporting or opposing the use of closed luer access devices to prevent CLABSI. 
Antimicrobial lock solutions (ALS) containing antibiotics (vancomycin, minocycline, gentamicin, cefazolin, and cefotaxim alone or in combination with gentamicin) decrease CLABSI rates in HD patients. Studies have shown that ALS containing gentamicin were more efficient than those without, but there is concern for publication bias. Other lock solutions with antimicrobial properties, such as citrate- and taurolidine-containing locks have showed mixed results regarding the impact on CLABSI incidence in dialysis patients. A novel catheter lock solution containing $7.0 \%$ sodium citrate, $0.15 \%$ methylene blue, $0.15 \%$ methylparaben, and $0.015 \%$ propylparaben (C-MB-P) was superior to standard heparin lock in a randomized controlled trial of $407 \mathrm{HD}$ patients [24]. Thrombolytic locking solution has also shown promise in reducing bacteremias. The use of recombinant tissue plasminogen activator (rt-PA) substituting heparin at the midweek dialysis session significantly reduced the incidence of catheter malfunction and bacteremia [25]. When used for prevention, ALS significantly lowers CLABSI risk. In our opinion, antibiotics used in the treatment of systemic infections should not be routinely used for prevention purposes in form of ALS due to the risk of antimicrobial resistance development. In contrast, citrate, CMB-P and rt-PA are not used for infection therapy and are therefore valuable alternatives. Nevertheless, the safety, applicability and cost of the latter two strategies still require further studies.

\section{Bundles}

The implementation of bundles containing various key interventions for infection prevention as discussed earlier has been advocated by the CDC initiative for reducing CLABSI in HD patients (table 2). The implementation of these key interventions or select elements thereof in a bundled approach has been shown to reduce CLABSI rates by $21-100 \%[5,26,27]$.

\section{Prevention and Surveillance at a Facility Level}

As mentioned above, prevention and management of CLABSIs in dialysis patients requires a multidisciplinary effort as suggested by the $\mathrm{CDC}$ core interventions. The most effective intervention for reducing CLABSIs is avoiding the placement of a CVC. The implementation of a multidisciplinary CVC team or designating a vascular access nurse in conjunction with evidence-based guide- 
lines has multiple benefits; but when it pertains to prevention, it has been shown to achieve the following goals $[28,29]$ :

- Increase the use of AVF as the first vascular access

- Reduce the total number of CVC days

- Reduce the rate of CVC loss due to infection

- Decrease the rate of treatment failure in CLABSI

- Lower the rate of death due to sepsis.

In order to track catheter infections over time and correlate them with preventive measures, the facility must perform CLABSI surveillance. Currently, all outpatient HD facilities in the US are required to conduct monthly surveillance using the NHSN Dialysis Event Manual (available at: http://www.cdc.gov/nhsn/ dialysis/dialysis-event.html). The facility has 60 days to submit the surveillance report with data about the three types of dialysis events: (1) intravenous antimicrobial starts - all intravenous antibiotics and antifungals; (2) positive blood culture, categorized by source as vascular access, other source or contaminant; (3) pus, redness, or increased swelling at the vascular access site.

Surveillance and prevention of drug-resistant organisms (DRO) such as MRSA and vancomycin-resistant Enterococcus (VRE) are still an unresolved issue without specific recommendations from the Infectious Diseases Society of America (IDSA) or European Renal Best Practice (ERBP). MRSA colonization in a high-prevalence country was found to be $2-4 \%$ in HD patients and $4-6 \%$ in dialysis unit personnel. Patients that require HD and are MRSA carriers have a 2.5-fold increase in all-cause mortality [30]. Decolonization techniques including nasal mupirocin and whole body chlorhexidine wash may achieve elimination of Staphylococcus and a risk reduction for major infections in the short term, but development of resistance has been documented. VRE colonization prevalence in HD patients can be up to 3-9\%, depending on geographical region. Of note, there is a significant potential for MRSA and VRE transmissions between HD patients in the dialysis setting [31]. The unique situation of dialysis patients (relative immunosuppression, frequent exposure to antibiotics, and repeated exposure to health care settings) exposes them to multiple opportunities to acquire DROs outside the dialysis setting and introduce it to the unit. The transmission dynamics of a dialysis unit are complex and involve large open rooms, shared treatment environment, and contact with multiple inanimate surfaces in common areas. Contact isolation of known DRO-colonized or -infected patients is difficult to achieve in outpatient HD centers, but effective when correctly implemented. Organizational strategies from other industries such as Toyota production systems (TPS) and positive deviance (PD) are being studied and have demonstrated a reduction of MRSA colonization, infection and BSI rates in a dialysis setting [32]. TPS involves the continuous identification of 
problem processes (e.g., inconveniently located alcohol dispensers for hand hygiene) and the standardization of solutions (e.g., placement of dispensers to optimize access). PD is a systems and behavior change strategy that recognizes exceptional progress or 'positive deviants' in a certain process (e.g., a specific nursing unit that achieved $100 \%$ hand hygiene rates) by studying their process and sharing it with the rest of the organization.

\section{Management of BSIs}

Recommendations for diagnosis and management of CLABSIs associated with HD catheters are based on IDSA [33] and ERBP [3] guidelines. Local signs may include erythema, warmth, swelling, tenderness, and purulent discharge, and may be found both in exit site infections and in BSIs, although the absence of local signs does not rule out bacteremia. The diagnosis of HD catheter-associated BSIs rests on blood culturing just like it does for CLABSIs outside the dialysis unit. If CLABSI is suspected (e.g., fever, sepsis, purulent discharge from the catheter exit site), at least two sets of blood cultures should be obtained, ideally pairing a catheter culture with another one drawn peripherally. If a fistula has yet to be created, peripheral cultures in that extremity may not be in order. It is recommended to apply a disinfectant to both catheter hub and skin before drawing these cultures. Also, intravenous antibiotics should be held until cultures have been taken. Common pathogens are Staphylococcus aureus, coagulase-negative staphylococci, and to a lesser degree, Gram-negative bacteria, enterococci and Candida. The formation of biofilm plays a major role in the pathophysiology and treatment of CLABSIs. In patients with bacteremia, the outer surface of the extravascular segment of the tunneled dialysis catheters has the thickest measured biofilm and is most likely to have an organism cultured [34]. The presence and location of the biofilm contributes to the need for catheter removal in certain infections. The management of CLABSIs is summarized in the flowchart (fig. 2). Infections are considered 'complicated' if any of the characteristics in table 3 is present.

Empiric therapy should be tailored to each institution's resistance profile, but usually will include vancomycin (or cefazolin if low prevalence of MRSA) plus another antibiotic with Gram-negative coverage (e.g., third-generation cephalosporin, carbapenem, or $\beta$-lactam/ $\beta$-lactamase inhibitor). If methicillin-susceptible S. aureus is found as the causative pathogen, then cefazolin or flucloxacillin/oxacillin should be the treatment of choice. In general, we prefer antibiotics that need to be administered after dialysis only. The most commonly utilized postdialysis antibiotic regimens include vancomycin, teicoplanin, cefazolin, 


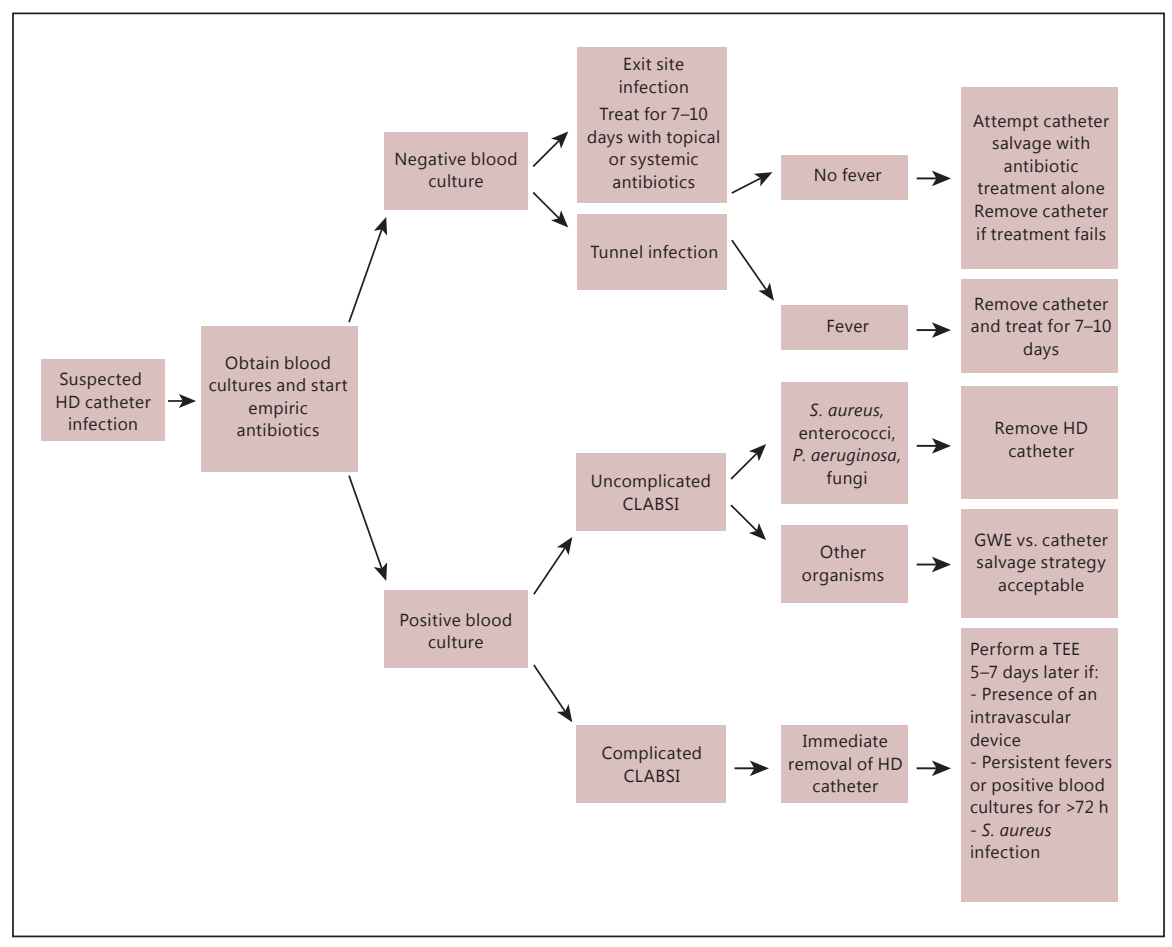

Fig. 2. Treatment algorithm for suspected HD catheter infection. TEE = Transesophageal echocardiography; GWE = guidewire exchange.

Table 3. The presence of any of these criteria should prompt the classification of a CLABSI as 'complicated CLABSI' and it should be managed accordingly

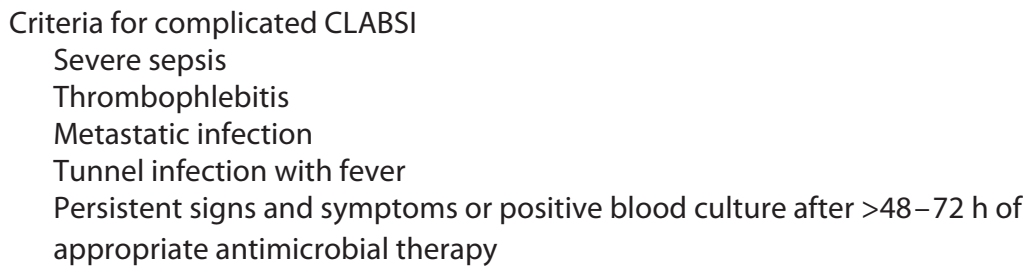

ceftazidime and daptomycin. The duration of antibiotic therapy for HD catheter infections is summarized in table 4 .

A frequently encountered clinical scenario in CLABSIs with HD catheters is the decision to perform a guide wire exchange (GWE) versus changing the access site. Immediate catheter removal is preferable in complicated infections. 
Table 4. Duration of antibiotic treatment for CLABSI after blood cultures are negative

Tunnel infection and negative blood culture

$7-10$ days

Uncomplicated CLABSI

$2-3$ weeks

Uncomplicated CLABSI with S. aureus

CLABSI with positive blood culture $>48-72 \mathrm{~h}$ after catheter removal

4 weeks $^{\mathrm{a}}$

6 weeks

CLABSI with metastatic infection

6 weeks

CLABSI and osteomyelitis

8 weeks

a $2-3$ weeks should suffice if transesophageal echocardiogram fails to show vegetations.

Performing GWE is acceptable with uncomplicated CLABSIs or when other access sites are unavailable. If clinical symptoms resolve within 2-3 days and there is no evidence of metastatic infection, GWE is associated with comparable cure rates to those encountered after immediate removal and delayed placement of a new catheter. If catheter removal or GWE is deemed unnecessary, undesirable or impossible, catheter salvage with antibiotic lock solution along with systemic antibiotics can be considered. In our personal experience, only a small number of dialysis patients qualify for lock therapy. Also, the logistics of administering lock therapy have to be considered. Both GWE and catheter salvage strategies are associated with (unacceptably) high treatment failure rate when S. aureus, Pseudomonas aeruginosa, enterococci or fungi are involved. If a GWE or catheter salvage strategy is chosen but the patient has ongoing symptoms for $>72 \mathrm{~h}$ or blood cultures are persistently positive, the catheter has to be removed. Surveillance blood cultures should be obtained one week after completion of antibiotic therapy in the setting of GWE or catheter salvage; if positive, catheter removal is indicated.

Infections of AVF are less frequent than CLABSI, and usually occur at the cannulation site. Vascular surgeons should evaluate the AVF to determine the need for surgical resection. Regardless, these infections should be treated for 6 weeks, in analogy to subacute bacterial endocarditis. Antibiotic therapy should be guided by culture results and resistance profiles. AVG infections usually require surgical revision with either partial, subtotal or total excision of the graft.

\section{Disclosure Statement}

A.S. and S.T. have no conflict of interest to report. Over the past 5 years, J.M. has been supported by the NIH CTSA (UL1RR024992) and is a recipient of a KL2 career development award (KL2RR024994). Via the NIH NCATS, he received support from the Building Interdisciplinary Research Careers in Women's Health program, a career develop- 
ment award (5K12HD001459-13). Currently, he is the section leader for a subproject of the CDC Prevention Epicenters Program grant (U54 CK000162; PI: Fraser). In addition, J.M. received funding from the Barnes-Jewish Hospital Patient Safety \& Quality Fellowship Program and a research grant from the Barnes-Jewish Hospital Foundation \& Washington University's Institute for Clinical and Translational Science. He has received funding for speaking at and attending an international conference from Gilead Sciences, Switzerland.

\section{References}

1 Umscheid CA, et al: Estimating the proportion of healthcare-associated infections that are reasonably preventable and the related mortality and costs. Infect Control Hosp Epidemiol 2011;32:101-114.

2 O'Grady NP, et al: Guidelines for the prevention of intravascular catheter-related infections. Clin Infect Dis 2011;52:e162-e193.

$\checkmark 3$ Vanholder R, et al: Diagnosis, prevention and treatment of haemodialysis catheter-related bloodstream infections (CRBSI): a position statement of European Renal Best Practice (ERBP). NDT Plus 2010;3:234-246.

4 Pronovost P, et al: An intervention to decrease catheter-related bloodstream infections in the ICU. N Engl J Med 2006;355: 2725-2732.

5 Patel PR, et al: Bloodstream infection rates in outpatient hemodialysis facilities participating in a collaborative prevention effort: a quality improvement report. Am J Kidney Dis 2013;62:322-330.

6 Klevens RM, et al: Dialysis Surveillance Report: National Healthcare Safety Network (NHSN)-data summary for 2006. Semin Dial 2008;21:24-28.

7 Camins BC: Prevention and treatment of hemodialysis-related bloodstream infections. Semin Dial 2013;26:476-481.

8 Yokoe DS, et al: A compendium of strategies to prevent healthcare-associated infections in acute care hospitals. Infect Control Hosp Epidemiol 2008;29(suppl 1):S12-S21.

9 Centers for Disease Control and Prevention: CDC Approach to BSI Prevention in Dialysis Facilities (i.e., the Core Interventions for Dialysis Bloodstream Infection (BSI) Prevention). 2013. http://www.cdc.gov/dialysis/prevention-tools/core-interventions.html.
10 Cheng SY, et al: Evaluation of inpatient multidisciplinary palliative care unit on terminally ill cancer patients from providers' perspectives: a propensity score analysis. Jpn J Clin Oncol 2013;43:161-169.

11 Boyce JM, Pittet D: Guideline for Hand Hygiene in Health-Care Settings: recommendations of the Healthcare Infection Control Practices Advisory Committee and the HICPAC/SHEA/APIC/IDSA Hand Hygiene Task Force. Infect Control Hosp Epidemiol 2002;23(12 suppl):S3-S40.

$\checkmark 12$ Lemaire X, et al: Analysis of risk factors for catheter-related bacteremia in 2000 permanent dual catheters for hemodialysis. Blood Purif 2009;28:21-28.

13 Chatzinikolaou I, et al: Antibiotic-coated hemodialysis catheters for the prevention of vascular catheter-related infections: a prospective, randomized study. Am J Med 2003; 115:352-357.

14 Maiwald M, Chan ES: The forgotten role of alcohol: a systematic review and meta-analysis of the clinical efficacy and perceived role of chlorhexidine in skin antisepsis. PLoS One 2012;7:e44277.

15 Oliver MJ, et al: Risk of bacteremia from temporary hemodialysis catheters by site of insertion and duration of use: a prospective study. Kidney Int 2000;58:2543-2545.

16 Timsit JF, et al: Chlorhexidine-impregnated sponges and less frequent dressing changes for prevention of catheter-related infections in critically ill adults: a randomized controlled trial. JAMA 2009;301:1231-1241.

17 Camins BC, et al: A crossover intervention trial evaluating the efficacy of a chlorhexidine-impregnated sponge in reducing catheter-related bloodstream infections among patients undergoing hemodialysis. Infect Control Hosp Epidemiol 2010;31:1118-1123. 
18 McCann M, Moore ZE: Interventions for preventing infectious complications in haemodialysis patients with central venous catheters. Cochrane Database Syst Rev 2010; CD006894.

19 Lok CE, et al: Hemodialysis infection prevention with polysporin ointment. J Am Soc Nephrol 2003;14:169-179.

20 Levin A, et al: Prevention of hemodialysis subclavian vein catheter infections by topical povidone-iodine. Kidney Int 1991;40:934938.

21 Simmons S, Bryson C, Porter S: 'Scrub the hub': cleaning duration and reduction in bacterial load on central venous catheters. Crit Care Nurs Q 2011;34:31-35.

22 Maragakis LL, et al: Increased catheter-related bloodstream infection rates after the introduction of a new mechanical valve intravenous access port. Infect Control Hosp Epidemiol 2006;27:67-70.

23 Jarvis WR, et al: Health care-associated bloodstream infections associated with negative- or positive-pressure or displacement mechanical valve needleless connectors. Clin Infect Dis 2009;49:1821-1827.

24 Maki DG, et al: A novel antimicrobial and antithrombotic lock solution for hemodialysis catheters: a multi-center, controlled, randomized trial. Crit Care Med 2011;39:613620.

25 Hemmelgarn BR, et al: Prevention of dialysis catheter malfunction with recombinant tissue plasminogen activator. N Engl J Med 2011; 364:303-312

26 Rosenblum A, et al: Hemodialysis catheter care strategies: a cluster-randomized quality improvement initiative. Am J Kidney Dis 2014;63:259-267.
27 Bren V, et al: Getting to zero: outpatient hemodialysis catheter-associated bloodstream infections; in Fifth Decennial International Conference on Healthcare-Associated Infections, Atlanta, March 2010.

28 Polkinghorne KR, Seneviratne M, Kerr PG: Effect of a vascular access nurse coordinator to reduce central venous catheter use in incident hemodialysis patients: a quality improvement report. Am J Kidney Dis 2009;53: 99-106.

29 Mokrzycki MH, et al: An interventional controlled trial comparing 2 management models for the treatment of tunneled cuffed catheter bacteremia: a collaborative team model versus usual physician-managed care. Am J Kidney Dis 2006;48:587-595.

30 Lai CF, et al: Nasal carriage of methicillinresistant Staphylococcus aureus is associated with higher all-cause mortality in hemodialysis patients. Clin J Am Soc Nephrol 2011;6: 167-174.

31 Lee SC, et al: An outbreak of methicillin-resistant Staphylococcus aureus infections related to central venous catheters for hemodialysis. Infect Control Hosp Epidemiol 2004; 25:678-684.

32 Ellingson K, et al: Sustained reduction in the clinical incidence of methicillin-resistant Staphylococcus aureus colonization or infection associated with a multifaceted infection control intervention. Infect Control Hosp Epidemiol 2011;32:1-8.

33 Mermel LA, et al: Clinical practice guidelines for the diagnosis and management of intravascular catheter-related infection: 2009 Update by the Infectious Diseases Society of America. Clin Infect Dis 2009;49:1-45.

34 Ramanathan V, et al: Characteristics of biofilm on tunneled cuffed hemodialysis catheters in the presence and absence of clinical infection. Am J Kidney Dis 2012;60:976-982.

Jonas Marschall, MD

Department of Infectious Diseases

Bern University Hospital

Friedbühlstrasse 51, CH-3010 Bern (Switzerland)

E-Mail jonas.marschall@insel.ch 